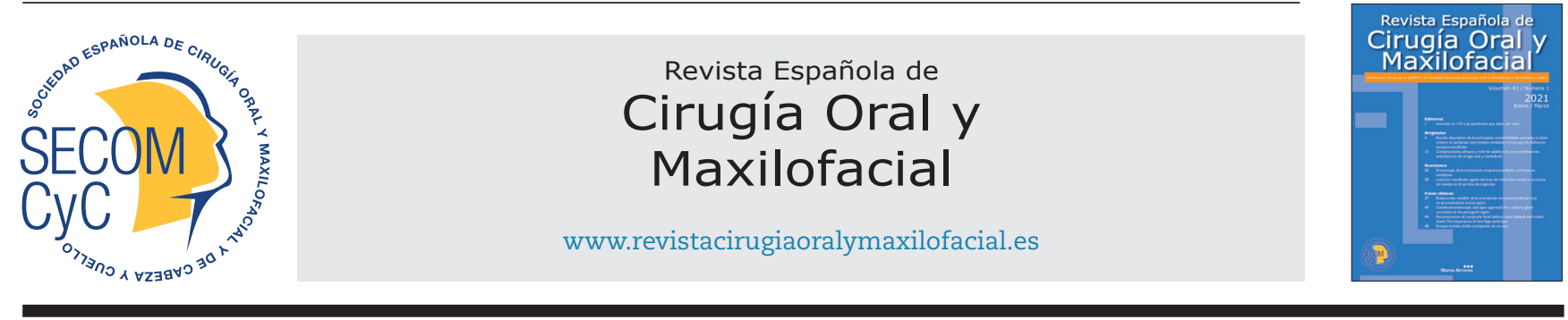

\title{
Original
}

\section{Estudio descriptivo de las principales comorbilidades asociadas al dolor crónico en pacientes intervenidos mediante artroscopia de disfunción temporomandibular}

\author{
Martín Fernández-Ferro ${ }^{1,2^{*}}$, Jacinto Fernández-Sanromán ${ }^{1,2}$, Aroa Gaspar-Barredo², \\ Alberto Costas-López ${ }^{1}$, Annahys López-Betancourt ${ }^{1}$, Pablo López-Fernández ${ }^{1}$, \\ Verónica Fernández-González ${ }^{1}$ y María Loreto Vidal-Castro ${ }^{3}$
}

${ }^{1}$ Servicio de Cirugía Oral y Maxilofacial. Hospital Povisa (Ribera Salud). Vigo, Pontevedra, España. ${ }^{2} E U E$ Povisa. Universidad de Vigo, Pontevedra, España. ${ }^{3}$ Unidad del Dolor. Servicio de Anestesiología y Reanimación. Hospital Povisa (Ribera Salud). Vigo, Pontevedra, España

INFORMACIÓN DEL ARTÍCULO

Historia del artículo:

Recibido: 28 de julio de 2020

Aceptado: 10 de enero de 2021

Palabras clave:

ATM, disfunción

temporomandibular, comorbilidad, migraña, fibromialgia, trastorno de ansiedad, depresión.

\section{R E S U M E N}

\begin{abstract}
Objetivos: Describir y analizar la posible relación entre las características clínicas y radiológicas de los pacientes intervenidos de una disfunción temporomandibular (DTM) y la presencia asociada de las principales comorbilidades vinculadas al dolor crónico orofacial según la literatura; el trastorno de ansiedad, la depresión mayor, la migraña y la fibromialgia.

Material y métodos: Se realizó un estudio retrospectivo y descriptivo de pacientes diagnosticados e intervenidos mediante artroscopia de una DTM de predominio articular entre los años 2006-2016. Distribuidos en dos grupos en función de la presencia de las comorbilidades descritas. Se realizó un análisis y descripción de las principales variables de exploración clínicas y radiológicas como el dolor (EVA), la apertura oral y la presencia de ruidos articulares, así como del desplazamiento discal, la presencia de cambios degenerativos, el componente miofascial y los estadios de Wilkes. Estas variables fueron evaluadas de forma preoperatoria. Resultados: Un total de 280 pacientes fueron incluidos en el estudio distribuidos en 2 grupos, formados mayoritariamente por mujeres (92,14 \%) con una media de edad de 38,6 años. Las comorbilidades más frecuentemente observadas fueron el trastorno de ansiedad (42 \%), la asociación entre ansiedad y depresión (22,7%) y la depresión (11,4%). Se describió una peor situación clínica en el grupo de pacientes con comorbilidades asociadas, con significación estadística en las variables sexo, dolor, ruidos articulares y estadios de Wilkes Avanzados (IV-V). Conclusiones: Según nuestro trabajo la mayoría de los pacientes intervenidos de una DTM crónica mediante artroscopia presentaban alguna comorbilidad vinculada al dolor crónico, destacando el trastorno de ansiedad. En este grupo de pacientes se observó además una peor situación clínica y funcional especialmente significativa en cuanto al dolor.
\end{abstract}

\footnotetext{
${ }^{*}$ Autor para correspondencia:

Correo electrónico: mfernandezf@povisa.es (Martín Fernández-Ferro).

DOI: 10.20986/recom.2021.1193/2020
}

1130-0558/C 2021 SECOM. Publicado por Inspira Network. Este es un artículo Open Access bajo la licencia CC BY-NC-ND (http:// creativecommons.org/licenses/by-nc-nd/4.0/). 


\section{Descriptive study of the main comorbidities associated with chronic pain in patients with temporomandibular dysfunction artroscopy}

\section{A B S T R A C T}

Keywords:

TMJ, temporomandibular dysfunction, comorbidity, migraine, fibromyalgia, anxiety disorder, major depression.

\begin{abstract}
Objectives: Describe and analyze the possible relationship between the clinical and radiological characteristics of patients on temporomandibular dysfunction (TMD) artroscopy and the associated presence of the main comorbidities related to chronic orofacial pain according to the literature; anxiety disorder, major depression, migraine, and fibromyalgia.

Material and methods: A retrospective and descriptive study of patients diagnosed and operated by artroscopy on a predominantly joint TMD between the years 2006-2016 was carried out. Distributed into two groups based on the presence of the comorbidities described. An analysis and description of the main clinical and radiological examination variables such as pain (VAS), oral opening and the presence of joint noises, as well as disc displacement, the presence of degenerative changes, the myofascial component and the stages of Wilkes were performed. These variables were evaluated preoperatively.

Results: A total of 280 patients were included in the study distributed in 2 groups, formed mainly by women (92.14 \%) with a mean age of 38.6 years. The most frequently observed comorbidities were anxiety disorder (42\%), association between anxiety and depression $(22.7 \%)$ and depression (11.4\%). A worse clinical situation was described in the group of patients with associated comorbidities, with statistical significance in the variables sex, pain, joint noises, and advanced Wilkes stages (IV-V).

Conclusions: According to our study, most of the patients operated on chronic TMD by arthroscopy had some comorbidity related to chronic pain, highlighting the anxiety disorder. In this group of patients, a particularly significant worse clinical and functional situation was observed in terms of pain.
\end{abstract}

\section{INTRODUCCIÓN}

La disfunción temporomandibular (DTM) constituye una entidad clínica muy frecuente con una incidencia, según algunas publicaciones, en torno al 12-30 \% de la población gene$\mathrm{ral}^{1-4}$. Esta patología puede involucrar a la propia articulación temporomandibular (ATM), a la musculatura masticatoria o a una combinación de ambas ${ }^{1,4,5}$.

Entre las características clínicas más comunes destacan el dolor muscular y/o articular, la limitación en la movilidad mandibular (apertura oral, lateralidades y movimiento protusivo) y la presencia de ruidos articulares (clic o crepitación); algunos autores añaden otros síntomas como otalgia, rigidez, fatiga, cefalea o dolor cervical, que pueden aparecer tanto en reposo como en relación con la función mandibular ${ }^{1-5}$. Todos estos signos y síntomas se pueden describir de forma aislada o combinada y en distintos grados de intensidad, lo que crea una entidad clínica muy heterogénea.

La etiología de la DTM es multifactorial y compleja. Algunos autores la clasifican como de origen articular y/o muscular ${ }^{1,2,4}$. En lo que respecta al origen articular, lo más habitual es la presencia de una alteración estructural o mecánica relacionada con la posición del disco, pero también se describen, dentro de la propia articulación, cambios inflamatorios (como sinovitis) y cambios degenerativos ${ }^{5-7}$. Respecto al componente muscular se menciona el síndrome miofascial temporomandibular como una alteración primaria y funcional de la musculatura masticatoria, de los tejidos blandos asociados, ligamentos y fascias. Este síndrome se caracteriza fundamentalmente por dolor crónico orofacial, que puede ser espontáneo o asociado a la palpación de la musculatura, hipersensibilidad, dolor irradiado a otras regiones y alteración de la función muscular ${ }^{3,4,8,9}$.

En conjunto, la DTM se presenta habitualmente en pacientes jóvenes y de mediana edad, entre los 20 y 60 años, y es más frecuente en mujeres, de forma que en algunas series se estima un porcentaje de hasta el $90 \%$ en el sexo femenino ${ }^{2,4}$.

El tratamiento es habitualmente conservador mediante el uso de férulas de reposo, ajustes oclusales, diversas técnicas de rehabilitación y fisioterapia, dieta blanda, acupuntura y antiinflamatorios ${ }^{1,2,4}$. Sin embargo, cuando dicho tratamiento fracasa, se suele plantear algún tipo de tratamiento quirúrgico. Este tratamiento suele ser necesario en menos del $10 \%$ de los pacientes ${ }^{2,5,7}$. En este contexto está ampliamente aceptado el papel de la artroscopia, como un tratamiento mínimamente invasivo con elevadas tasas de éxito para casos sintomáticos de predominio articular. Este procedimiento quirúrgico está indicado en pacientes con un índice de dolor que le limita en sus actividades normales y/o existe una limitación de la función mandibular ${ }^{1,2,5,7}$.

La DTM constituye una entidad clínica amplia y compleja, que puede cronificarse en lo que respecta a los síntomas del dolor orofacial y alteración de la movilidad mandibular. Es por ello que esta patología está incluida en la "Clasificación Internacional de los Trastornos de Cefaleas" bajo el epígrafe "cefalea o dolor facial atribuido a disfunción temporomandibular", y 
más recientemente en la "Clasificación Internacional de Dolor Orofacial (ICOP)" como "dolor de la articulación temporomandibular y dolor miofascial orofacial"1,3,8.

En este sentido, se menciona en la literatura que la DTM crónica puede presentarse asociada e interactuar con otras afecciones vinculadas con el dolor crónico de la región orofacial, destacando por su frecuencia el trastorno de ansiedad, la depresión mayor, la fibromialgia y la migraña $a^{3,8-11}$.

Algunos autores describen que esta asociación no es puramente casual, sino que existen evidencias de una posible relación de comorbilidad, de manera que estas patologías "distintas" podrían participar en la evolución clínica de la DTM y ser en parte responsables de una mala gestión y, por lo tanto, de un mal pronóstico $3,8,10-13$.

Los mecanismos neuronales o bioquímicos relacionados con la fisiopatología del dolor crónico orofacial vinculados a la DTM crónica y a estas cuatro patologías son desconocidos y motivo de discusión ${ }^{10-12}$. Sin embargo, se reconoce la importancia que pueden tener múltiples factores biológicos (estructurales y/o funcionales), pero también factores psicológicos. Relacionados con estos últimos, son numerosas las referencias que vinculan a los trastornos del estado de ánimo (depresión) y de la ansiedad con una mayor intensidad de los síntomas relacionados con el dolor, su cronificación y una peor calidad de vida ${ }^{8,10,12}$. Por otro lado, también vinculadas a la génesis del dolor crónico se mencionan las cefaleas primarias (concretamente la migraña) y la fibromialgia. En ambos casos, su mecanismo de asociación al dolor crónico es complejo, y se cree que está relacionado con una mayor sensibilidad miofascial en las distintas regiones musculoesqueléticas, con especial atención a la musculatura masticatoria, en las regiones orofacial, craneal y cervical ${ }^{9,10,12-14}$.

Hasta el momento actual no se han publicado estudios que investiguen la posible relación entre estas patologías crónicas y los pacientes con DTM en los que se ha realizado un procedimiento de artroscopia.

Es por ello que el principal objetivo de nuestro trabajo es describir y discutir la posible relación entre la presencia de estas comorbilidades, relacionadas con el dolor crónico orofacial, y los datos clínicos y radiológicos de pacientes seleccionados diagnosticados de una DTM que no responde al tratamiento conservador y en los que se ha indicado un procedimiento de artroscopia.

\section{MATERIAL Y MÉTODO}

El siguiente estudio retrospectivo descriptivo fue realizado de acuerdo a los estándares internaciones de calidad de los estudios de investigación clínica, como la Declaración de Helsinki en su versión revisada (Fortaleza, Brasil 2013) y el protocolo de trabajo ha sido aprobado por el Comité de Ética de Investigación Clínica de Galicia (Código de Registro: 2017/463) que es de referencia para nuestro centro el Hospital Povisa (Ribera Salud), Vigo, Pontevedra, España. Todos los pacientes cuentan con consentimiento informado.

Una cohorte de 280 pacientes fue incluida en el estudio desde 2006-2016. Se realizó una revisión exhaustiva de los antecedentes personales de cada paciente, lo que permitió establecer dos grupos, por un lado los casos en los que se diagnosticó algunas de las comorbilidades objetivo principal del estudio (trastorno de ansiedad, depresión mayor, fibromialgia y migraña) y, por otro lado, el resto de los casos que están formados por pacientes en los que no se pudo establecer esta asociación. Debemos de añadir que solo se tuvieron en cuenta los diagnósticos crónicos de las patologías comórbidas realizados por especialistas en Psiquiatría, Reumatología y Neurología.

En lo que respecta a la DTM, todos los pacientes fueron evaluados de acuerdo con los criterios diagnósticos para la disfunción temporomandibular (RDC/DTM) ${ }^{15}$. En todos los casos se realizó un estudio radiológico mediante resonancia magnética (RNM) en dinámica (Achieva 1.5-T, versión 3.2 nivel 3; Philips Medical Systems, Holanda). Los criterios de RNM que definen esta disfunción articular se refieren principalmente a la posición del disco, a la presencia de cambios degenerativos y de líquido intrarticular o derrame ${ }^{6}$. Todos los pacientes incluidos en este estudio tenían síntomas clínicos de disfunción temporomandibular crónica de origen articular que no respondieron al tratamiento conservador, que incluyó el uso de antinflamatorios no esteroideos, fisioterapia y férulas oclusales, por un periodo de tiempo superior a 6 meses.

En todos los casos se indicó y realizó un procedimiento de artroscopia operativa con anestesia general, de forma ambulatoria y por el mismo equipo quirúrgico. La técnica artroscópica se realizó con una entrada de doble portal utilizando dos cánulas de 2,3 mm y un artroscopio de Dyonics ${ }^{\circledR}$ de 1,9 mm con ángulo de 30" (Smith \& Nephew, Melbourne, Australia). Se realizó una exploración del espacio articular, lisis y lavado y mediante la segunda cánula se introdujo el dispositivo de coblación (ArthroCare ${ }^{\circledR}$ Reflex Ultra ${ }^{\circledR} 45$, ArthroCare ${ }^{\circledR}$, Sunnyvale, CA, EE. UU.). Según el caso, se realizó la liberación del disco mediante capsulotomía y miotomía (músculo pterigoideo lateral), escarificación del tejido retrodiscal, vaporización de las áreas de sinovitis y liberación de las adherencias ${ }^{7}$. Durante el postoperatorio todos los pacientes recibieron el mismo tratamiento y recomendaciones.

Los criterios de inclusión fueron: pacientes con síntomas clínicos de patología temporomandibular unilateral, con estudio de RNM y con indicación de artroscopia. Los criterios de exclusión fueron: disfunción bilateral, patología articular relacionada con deformidades mecánicas, secuelas de traumatismos, enfermedades autoinmunes, enfermedades infecciosas, infiltraciones o tratamiento quirúrgico previo de la ATM.

Se recogieron los datos de las siguientes variables de exploración clínicas preoperatorias en ambos grupos (grupo con y sin comorbilidades asociadas): edad y sexo, dolor (medido mediante escala visual analógica [EVA] con valores de entre 0-10), apertura máxima interincisal (medida como la distancia máxima entre los incisivos superiores e inferiores en $\mathrm{mm}$ ), presencia de ruidos articulares (sí/no), y presencia de componente miofascial asociado (sí/no). Los criterios que se establecieron para el diagnóstico de un síndrome miofascial asociado fueron clínicos y hacen referencia, fundamentalmente, al dolor a la palpación de la musculatura masticatoria y también se tuvieron en cuenta la presencia de puntos gatillo miofasciales o bandas tensas musculares y su repercusión en funciones básicas como comer o hablar ${ }^{4,9}$.

Como variables radiológicas se recogieron: la posición del disco distinguiendo entre posición normal, desplazamiento discal con reducción (DDCR) y sin reducción (DDSR), presencia de cambios degenerativos (sí/no), presencia de derrame intrarticular (sí/no) y, por último, se incluyó la clasificación de los 
pacientes según la escala de Wilkes, que permite clasificar los casos según la severidad de los datos clínicas y radiológicas distinguiendo entre estadios tempranos (II-III) y avanzados (IV-V) ${ }^{5}$.

Se realizó un estudio estadístico descriptivo a partir de los datos recogidos de los antecedentes médicos, datos de exploración clínica y radiológicos preoperatorios de los pacientes intervenidos de DTM mediante artroscopia en el periodo de tiempo señalado. Se describió mediante la presentación de medias y desviaciones estándar (DS) las variables continuas, y números y porcentajes para las variables categóricas. Para revelar posibles factores de confusión sociodemográficos se compararon las diferencias entre los participantes, con y sin comorbilidades. Las diferencias entre las variables sociodemográficas se comprobaron utilizando T-test para variables continuas y Chi cuadrado para variables categóricas. Los distintos grupos y variables se compararon con la prueba de significación para medias, prueba U de Mann-Whitney como alternativa a la prueba $\mathrm{T}$ de Student, cuando la variable dependiente se midió en intervalos y las diferencias de los grupos respecto a las variables categóricas fueron evaluadas mediante la prueba de Chi cuadrado. El nivel de significación se estableció como $p<0,05$. Se empleó el Paquete Estadístico IBM SPSS Statistic en su versión 25.0. Armonk, NY, EE. UU.

\section{RESULTADOS}

Una cohorte total de 280 pacientes fue incluida en el estudio, que se dividió en 2 grupos. Uno de los grupos constituyó el $58,21 \%$ de los casos $(n=163)$ y estuvo formado por pacientes con DTM que presentaron alguna de las patologías comórbidas estudiadas (grupo con comorbilidades), y el otro grupo supuso el $41,78 \%$ de los casos ( $n=117$ ) y estuvo compuesto por pacientes que no presentaron ninguna comorbilidad en sus antecedentes médicos (grupo sin comorbilidades).

La patología comórbida más frecuentemente observada fue el trastorno de ansiedad con un $42 \%$, es decir, el $42 \%$ de los casos de este grupo presentaba al menos un trastorno de ansiedad, seguida del trastorno de depresión mayor con un 31 \%, en tercer lugar, la fibromialgia con un $17 \%$ y por último la migraña con un $10 \%$ (Figura 1).

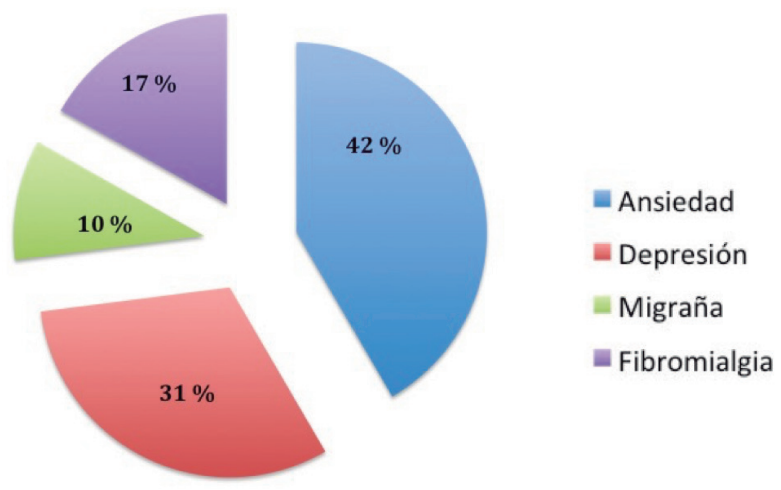

Figura 1. Descripción en porcentaje de cada una de las comorbilidades presentes en el estudio.
Sin embargo, debemos de tener en cuenta que en muchos casos se describieron varias comorbilidades en un mismo paciente, lo que dio lugar a distintas combinaciones de patologías en el 44,17 \% de los casos ( $n=72$ ). De entre estas combinaciones destacó por su frecuencia la asociación del trastorno de ansiedad y depresión mayor con un 22,7 \% ( $n=37)$ y el trastorno de ansiedad, depresión mayor y fibromialgia con un $10,42 \%$ ( $\mathrm{n}=17$ ) (Tabla I).

El 92,14 \% de los casos fueron mujeres $(n=258)$ y el $7,86 \%$ varones $(n=22)$, siendo la proporción 11:1. En el grupo de pacientes con comorbilidades las mujeres suponen el 95,70\% ( $n=156)$, y dentro del grupo formado por únicamente por mujeres destacó la presencia del trastorno de ansiedad como el más frecuente $(69,87 \%)$, seguido de la fibromialgia (12,82 \%) y la presencia de un trastorno de depresión (10,89 \%). En este grupo, los varones fueron el 4,29 \% ( $n=7)$ y destacó la depresión mayor como la principal comorbilidad (71,42 \%). En el estudio analítico se observó una diferencia estadísticamente significativa en lo que respecta a la variable sexo con un valor de $p=0,009$.

La edad media de los pacientes fue de 38,6 años, en el grupo con comorbilidades fue de 39 años y de 37 años para el grupo sin comorbilidades, en un rango de entre 18-74 años. El grupo de edad mayoritario, para ambos grupos, se situó entre los 35-65 años, $(53,93 \%, n=151)$, siendo los menores de 35 años el $41,78 \%(n=117)$.

Respecto a las principales variables de exploración clínicas de la disfunción temporomandibular observamos que el dolor medio (EVA) en el total de la muestra $(n=280)$ fue de 7,66 \pm 0,921 , destacando que el $62,50 \%(n=175)$ de los pacientes presentaron valores de entre 8-10. En el grupo de pacientes sin comorbilidades la media de dolor fue $7,48(\mathrm{n}=117)$ y la mayoría presentaron un valor de EVA de entre 5-7 (50,48 \%) frente al grupo con comorbilidades, donde la media fue de 7,79 ( $n=163$ ) y en este caso la mayoría presentaron un valor de EVA de entre 8-10 (63,43\%). En el estudio analítico se observó una diferencia significativa en relación con esta variable entre los grupos de estudio $p=0,004$ (Tabla II).

Tabla I. Descripción completa de cada una de las comorbilidades estudiadas de forma aislada e incluyendo las asociaciones.

\begin{tabular}{lcc}
\multicolumn{1}{c}{ Comorbilidades } & $\mathrm{n}=163$ & $\%$ \\
\hline Ansiedad & 44 & 27 \\
Depresión & 18 & 11,04 \\
Migraña & 10 & 6,13 \\
Fibromialgia & 19 & 11,68 \\
Ansiedad-Depresión & 37 & 22,7 \\
Ansiedad-Migraña & 6 & 3,68 \\
Ansiedad-Depresión-Migraña & 6 & 3,68 \\
Ansiedad-Depresión-Fibromialgia & 17 & 10,42 \\
Ansiedad-Depresión-Migraña- & 2 & 1,22 \\
Fibromialgia & 2 & 1,22 \\
Depresión-Fibromialgia & 1 & 0,61 \\
Depresión-Migraña-Fibromialgia & 1 & 0,61 \\
Migraña-Fibromialgia &
\end{tabular}


Tabla II. Descripción de las variables recogidas en el estudio.

\begin{tabular}{|c|c|c|c|}
\hline Variables & $\begin{array}{l}\text { Sin Comorbilidades } \\
(\mathrm{n}=117)\end{array}$ & $\begin{array}{l}\text { Con comorbilidades } \\
\text { ( } \mathrm{n}=163)\end{array}$ & $p$ valor \\
\hline Edad media (DS) & $37,32( \pm 13,4)$ & $39,28( \pm 12,3)$ & 0,214 \\
\hline Género n, (\%) & & & 0,009 \\
\hline Femenino & $102(87,17 \%)$ & $156(95,70 \%)$ & \\
\hline Masculino & $15(12,82 \%)$ & $7(4,29 \%)$ & \\
\hline Dolor medio, EVA (DS) & $7,48( \pm 0,93)$ & $7,79( \pm 0,87)$ & 0,004 \\
\hline Componente miofascial, n (\%) & $78(66,6 \%)$ & $117(71,78 \%)$ & 0,359 \\
\hline Apertura máxima mm (DS) & $28,68( \pm 5,07)$ & $28,56( \pm 4,32)$ & 0,84 \\
\hline Ruidos articulares, n (\%) & $33(28,20 \%)$ & $83(50,92 \%)$ & 0,011 \\
\hline Posición discal según $\mathrm{RNM}, \mathrm{n}$ (\%) & & & 0,958 \\
\hline Normal & $10(8,54 \%)$ & $7(4,29 \%)$ & \\
\hline DDCR & $16(13,67 \%)$ & $17(10,42 \%)$ & \\
\hline DDSR & $91(77,77 \%)$ & $139(85,27 \%)$ & \\
\hline Cambios degenerativos (OA) según RNM, n (\%) & $64(54,7 \%)$ & $106(65,03 \%)$ & 0,064 \\
\hline Derrame articular según RNM, n (\%) & $22(18,8 \%)$ & $32(19,63 \%)$ & 0,862 \\
\hline Estadios de Wilkes IV-V, n (\%) & $58(49,57 \%)$ & $107(65,64 \%)$ & 0,007 \\
\hline
\end{tabular}

La apertura media máxima interincisal $(\mathrm{mm})$ del total de la muestra fue de 28,61 \pm 4,64 (intervalo de confianza del $95 \%$ ). La mayoría de los casos $(66,79 \%, n=187)$ presentaron una apertura máxima comprendida entre 25-30 mm. Los pacientes sin comorbilidades presentaron una apertura media de $28,68 \mathrm{~mm}$ y en la mayoría de los casos $(56,60 \%, n=66)$ una apertura mayor de $30 \mathrm{~mm}$; en cambio, los pacientes con comorbilidades presentaron una apertura media de $28,56 \mathrm{~mm}$, destacando que la mayoría tuvieron una apertura comprendida entre $25-30 \mathrm{~mm}$ $(69,93 \%, n=114)$ e incluso el $11,65 \%(n=19)$ tuvo una apertura menor de $25 \mathrm{~mm}$.

En cuanto a los ruidos articulares, el 58,57 \% ( $\mathrm{n}=164)$ no presentaron ningún ruido, el 25,36 \% ( $n=71)$ presentaron un clic de apertura y el 16,07 \% ( $n=45)$ presentaron crepitación. Los pacientes sin comorbilidades no presentaron mayoritariamente ningún ruido $(71,79 \%, \mathrm{n}=84)$ y los pacientes con comorbilidades presentaron mayoritariamente algún ruido articular $(50,96 \%, \mathrm{n}=83)$, como clic de apertura $(30,67 \%, \mathrm{n}=50)$ o crepitación $(20,24 \%, n=33)$. En este caso también se objetivó una diferencia significativa entre ambos grupos $(p=0,011)$.

Por otro lado, en la mayoría de la muestra se observó una disfunción temporomandibular con componente miofascial asociado $(69,64 \%, n=195)$, de los cuales el 71,78 \% ( $n=117)$ presentaba alguna comorbilidad y el $66,6 \%(n=78)$ no presentaba comorbilidades.

Dentro de las principales variables radiológicas, la posición del disco fue normal en el 6,08 \% ( $n=17)$ y presentó algún grado de desplazamiento en el 93,92\% $(n=263)$ del total de la muestra. Destacamos sobre todo el desplazamiento discal anterior sin reducción (DDSR) que en el total de la muestra fue de 82,14\% ( $n=230)$. El grupo de pacientes con alguna comorbilidad presentaron mayoritariamente un DDSR $(85,27 \%, \mathrm{n}=139)$, así como el grupo de pacientes sin comorbilidad $(77,77 \%$, $\mathrm{n}=91)$. En lo que respecta a la morfología del componente óseo en la RNM en el total de la muestra, el 60,71 \% ( $n=170$ ) de los casos presentaron cambios degenerativos. En el grupo con comorbilidades, el $65,03 \%(n=106)$ presentaron cambios degenerativos y en el grupo sin comorbilidad solo el $54,7 \%$ ( $n=64)$ presentaron alguna alteración en la morfología ósea. En la mayoría de los casos $(80,71 \%, n=226)$ no se observó en la RNM la presencia de derrame intrarticular, siendo este ligeramente superior en el grupo con comorbilidades 19,63\% ( $\mathrm{n}=32$ ) (Tabla II).

Finalmente, en cuanto a la clasificación de los casos según la Escala de Wilkes, observamos que en el total de la muestra la mayoría presentaron un estadio avanzado (IV-V) (58,93 \%, $\mathrm{n}=165)$. En lo que respecta al grupo con comorbilidades, la mayoría de los casos $(65,64 \%, \mathrm{n}=107)$ presentaron también un estadio avanzado (IV-V) y en este caso, en el estudio analítico se observó, además, una diferencia estadísticamente significativa $(p=0,007)$ (Tabla II).

\section{DISCUSIÓN}

La mayoría de los autores coinciden al señalar que la patología temporomandibular constituye una entidad clínica muy frecuente, caracterizada por dolor en la ATM y/o musculatura masticatoria, lo que afecta al movimiento, a la función y, en general, a la calidad de vida de los pacientes, y se destaca además que es mucho más frecuente en mujeres ${ }^{1,4,13-15}$. A lo largo del tiempo se han sugerido diversos tratamientos para el manejo de esta patología, lo que sigue siendo un tema controvertido, y en el que se distinguen desde terapias más conservadoras hasta técnicas mínimamente invasivas como la artroscopia. Todos estos tratamientos tienen en común que están dirigidos a controlar o paliar estos síntomas específicos, con especial atención al dolor, que puede ser espontáneo o asociado a la función mandibular, y que con el tiempo puede llegar a cronificarse ${ }^{1-5,7}$. 
Son muy frecuentes las referencias en la literatura que describen de forma precisa las características clínicas de los pacientes con DTM, desde un punto de vista articular y muscular, y que hacen referencia fundamentalmente a lesiones estructurales y/o funcionales de la propia ATM y/o musculatura masticatoria ${ }^{1-5,7}$. Sin embargo, son muy escasos los trabajos en los que se realiza un análisis más profundo y amplio de los aspectos que pudieran estar involucrados en los mecanismos subyacentes del dolor crónico, característico en muchos de estos pacientes 8,10

En relación con esta idea, se menciona en la literatura la posible relación comórbida entre algunas entidades clínicas vinculadas al dolor crónico orofacial y la DTM $\mathrm{DT}^{10,14,16-18}$.

En nuestro trabajo, y coincidiendo con la mayoría de las series $^{1,5,8}$, la DTM se describió más frecuentemente en mujeres en ambos grupos (92,14\%), observando además una relación positiva entre el sexo femenino y la presencia de comorbilidades $(p=0,009)$. En la literatura se menciona que las mujeres sufren en mayor proporción diversas condiciones vinculadas al dolor crónico, como los trastornos de ansiedad, migraña o fibromialgia, y que esta mayor prevalencia, en algunos casos también significativa, puede estar relacionada con factores fisiológicos u hormonales, destacando el papel pronociceptivo de los estrógenos ${ }^{10-14}$. Entre los varones, estas patologías no son tan prevalentes, excepto los trastornos del estado de ánimo como la depresión ${ }^{12,19}$.

La edad media de los pacientes, en el total de la muestra, fue de 38,6 años, señalando que la mayoría de los casos se situaron entre los 35-45 años (53,93\%). También coincidiendo con la literatura, la DTM es más frecuente en pacientes jóvenes o de medida edad, con un pico de incidencia entre los 20 y 50 años $2,4,9$.

En lo que respecta a la distribución de las entidades comórbidas estudiadas, destacamos que este grupo de pacientes fue mayor $(58,21 \%, \mathrm{n}=163)$ respecto al grupo sin estas patologías $(41,78 \%, n=117)$. Por otro lado, observamos que la patología más frecuente fue el trastorno de ansiedad (42\%) y el trastorno de depresión (31 \%), así como la asociación de ambos (22,7 \%), seguido de la fibromialgia (17\%) y la migraña (10 \%). Dahan y cols. describen en su trabajo que el $62 \%$ de los casos de DTM presentaba alguna comorbilidad, fundamentalmente en mujeres (86,6\%) y con una edad media de 41 años, y resaltan que el trastorno de ansiedad, la depresión y la migraña fueron las patologías más prevalentes ${ }^{13}$. En otros estudios similares se menciona una asociación significativa entre la fibromialgia y la DTM, con una prevalencia elevada de entre el 33-97 \% según las series, y siendo más frecuente en mujeres de edad media. Esta elevada prevalencia se relaciona con la participación del sistema masticatorio entre los síntomas más comunes de las personas con fibromialgia, por lo que se piensa que la fibromialgia puede tener un papel desencadenante y predisponente para la cronificación del dolor orofacial en la $\mathrm{DTM}^{3,16-18}$. Ayouni y cols. enfatizan en su trabajo la idea de incluir los síntomas y signos clínicos característicos de la patología temporomandibular entre los criterios diagnósticos de la fibromialgia, dada la alta prevalencia de la DTM en los pacientes diagnosticados de fibromialgia, lo que podría tener importancia a la hora de establecer un diagnóstico precoz y aplicar el tratamiento más oportuno ${ }^{16}$.
En lo que respecta a las variables de exploración clínica, el síntoma más frecuente e importante fue el dolor, que será el principal síntoma vinculado a las comorbilidades descritas $^{3,17,19}$. En general, se considera que el dolor en la DTM puede tener dos componentes principales. Por un lado el dolor generado a nivel articular, relacionado con alteraciones estructurales o de posición del disco, que en algunos pacientes cursan sin dolor ${ }^{20}$ y que en otros el dolor puede llegar a cronificarse ${ }^{1,8}$, y por otro lado, el dolor de origen miogénico o miofascial ${ }^{9,17}$. Fernández-de-las-Peñas y cols. describen en su trabajo de revisión un metanálisis que incluyó un total de 3463 pacientes en el que señala que la prevalencia del dolor miofascial en la DTM fue del 45,3\%, mientras que el dolor vinculado al desplazamiento discal fue del 41,1 \% ${ }^{9}$. Estos autores añaden que hay evidencias de múltiples factores que se relacionan con complejos mecanismos de sensibilización central, mecanismos de inhibición, participación del sistema trigeminal e incluso factores psicológicos, que interactúan en el desarrollo, cronificación y relación del dolor crónico de la DTM con las entidades comórbidas ${ }^{9}$.

El dolor (EVA) en el grupo pacientes con comorbilidades asociadas tuvo un valor medio de 7,79, destacando que la mayoría de estos casos tuvieron valores elevados de dolor EVA 8-9 (63,43\%), respecto al grupo de pacientes sin comorbilidades, en los que la media de dolor fue de 7,48 y la mayoría de los casos presentaron un valor de EVA entre 5-7 (50,48 \%). En este caso, además, se objetivó una relación positiva respecto a los pacientes sin comorbilidades $(p=0,004)$.

Costa y cols. consideran en su trabajo que el dolor vinculado a la DTM, a la migraña y a la fibromialgia comparten mecanismos de sensibilización central y la participación del complejo neuronal trigémino-cervical, de forma que mediante mecanismos complejos, estímulos que en circunstancias normales serían inofensivos se interpretan de manera que producen una sensación abrumadora y prolongada de dolor, especialmente en cuanto a su intensidad y duración ${ }^{10}$. Por otro lado, también se menciona, una relación positiva vinculada a la presencia de ansiedad y/o depresión en relación con el dolor en estos pacientes. Se piensa que el papel de las interacciones entre los factores biológicos y psicológicos en la génesis del dolor orofacial es complejo, ya que actúan como neuromoduladores que pueden aumentar el estímulo responsable el dolor agudo empeorando, el pronóstico y favoreciendo la cronificación del mismo, especialmente cuando existe un componente miofascial asociado a la DTM ${ }^{19,21,22}$.

Son frecuentes las referencias en las que se señala que la relación entre los factores psicológicos asociados a la DTM puede incidir de forma negativa en el diagnóstico, evolución y planificación del tratamiento de estos pacientes ${ }^{11,13,22}$. Sin embargo también se menciona que la patología crónica dolorosa, entre la que se incluye a la DTM, puede por sí misma, generar una mayor susceptibilidad para el desarrollo de trastornos depresivos o ansiosos en estos pacientes ${ }^{23}$. Se hace referencia en la literatura a esta dualidad compleja del dolor crónico, que puede ser causa y/o consecuencia de estas patologías, e incluso que los procesos inflamatorios vinculados a este dolor crónico pueden alterar la respuesta a los tratamientos antidepresivos 8,23

La apertura oral media en el grupo de pacientes con comorbilidades fue de entre $25-30 \mathrm{~mm}$, o menor de $25 \mathrm{~mm}$ en el $72,34 \%$ 
de los casos, respecto al grupo sin comorbilidades en los que la mayoría presentó una apertura inicial superior a $30 \mathrm{~mm}$ (56,60\%). El grupo de pacientes con comorbilidad presentó mayoritariamente algún ruido articular (50,96\%) respecto a los pacientes sin comorbilidad, que mayoritariamente no presentaron ruidos; en este caso se observó además una diferencia significativa ( $p=0,011)$. Moreno-Fernández y cols. mencionan en su trabajo que la relación entre la fibromialgia y la DTM puede estar basada en una hipersensibilidad nociceptiva relacionada con el daño muscular, de forma que el estrés oxidativo puede causar hiperalgesia, fatiga, rigidez y discapacidad en la función mandibular ${ }^{3}$. Leblebici y cols. describen una relación significativa entre la DTM de origen articular y la fibromialgia, haciendo referencia a los ruidos articulares, al desplazamiento discal y a los cambios degenerativos, lo que favorece una mayor tendencia al dolor y a la discapacidad funcional ${ }^{17}$.

Respecto a la presencia del componente miofascial, este se observó en la mayoría de la muestra 69,64 \% (n=195) y el $71,78 \%(n=117)$ de los casos presentaba alguna comorbilidad.

La limitación en la apertura, los ruidos articulares y el componente miofascial se pueden relacionar con la discapacidad funcional vinculada a la DTM crónica. En la literatura se recoge el papel destacado de las comorbilidades en el desarrollo y mantenimiento de esta alteración funcional ${ }^{1,13,21,24}$. Se describe cómo la migraña y la fibromialgia aumentan la limitación y el deterioro funcional afectando a los movimientos mandibulares y a la apertura oral, favoreciendo la rigidez y la fatiga que repercute negativamente en funciones básicas como la masticación y fonación ${ }^{11,17,18}$. El trastorno de ansiedad y depresión se vinculan también con una mayor discapacidad física y psicológica, ya que favorecen los hábitos parafuncionales, la perpetuación del componente miofascial y la peor percepción en la calidad de vida ${ }^{22,25}$. Slade y cols., describen en su trabajo prospectivo sobre el dolor orofacial, que el componente miofascial asociado a la DTM es muy característico, predisponente y factor pronóstico, y aparece vinculado a otros factores "biopsicosociales", entre los que destaca la ansiedad y/o la depresión ${ }^{24}$.

En cuanto a las variables radiológicas, destacamos que ambos grupos presentaron mayoritariamente un DDSR (82,14\%) asociado a cambios degenerativos $(60,71 \%)$. Creemos que esta presencia mayoritaria de un DDSR y de cambios degenerativos entre los hallazgos de RNM está relacionada con el hecho de que la muestra analizada está formada por pacientes con DTM crónica y sintomática, con clara indicación de un procedimiento de artroscopia ${ }^{1,5-7}$.

Finalmente, respecto al Estadio de Wilkes, el grupo con comorbilidades presentó mayoritariamente un estadio avanzado (IV-V) 65,64\%, resultado una diferencia estadísticamente significativa $(p=0,007)$, respecto al grupo sin comorbilidades, hecho que relacionamos con el mayor deterioro clínico y funcional vinculado al grupo de pacientes con estas patologías asociadas $^{11,13,17}$.

Tras el análisis de los datos en el conjunto de la muestra estudiada, queremos destacar tres aspectos que se pueden correlacionar entre sí: en primer lugar la mayor presencia de comorbilidades en pacientes con DTM crónica de predominio articular con participación del componente miofascial y que no responden al tratamiento conservador, por otro lado que este grupo de pacientes con comorbilidades presentaron una situación clínica, funcional y radiológica claramente peor, a veces con una diferencia significativa respecto al grupo sin comorbilidades, y por último hacer hincapié en la idea que se enfatiza en la literatura respecto a que los pacientes con DTM vinculada a alguna de las comorbilidades descritas presentan mayor dificultad diagnóstica, peor evolución del proceso y peor pronóstico, lo que puede implicar un impacto negativo en el curso clínico de la disfunción temporomandibular y contribuir a cronificar los síntomas de dolor y discapacidad ${ }^{8,10,19}$.

Como principales limitaciones de nuestro trabajo destacamos que es posible que no se represente el perfil promedio de los pacientes diagnosticados de una DTM que presentan dolor agudo o autolimitado, sino más bien el de personas con DTM crónica. Por otro lado, se ha realizado únicamente un estudio descriptivo de las comorbilidades en conjunto y no desde un punto de vista más analítico e individualizando a cada una de ellas, y únicamente se han recogido aspectos relacionados con datos clínicos preoperatorios.

Por todo ello, creemos que el estudio de las comorbilidades vinculadas al dolor orofacial en relación a DTM puede tener gran interés en nuestra práctica clínica diaria, por lo que sería necesario realizar nuevos estudios más amplios y detallados. Estudiar y entender estas asociaciones, puede dar apoyo a un enfoque multidisciplinar más amplio, que permita una mayor calidad en la toma de decisiones en pacientes seleccionados con DTM.

\section{CONCLUSIONES}

1. Mayoritariamente los pacientes intervenidos de DTM crónica mediante artroscopia presentaron en sus antecedentes la presencia de alguna comorbilidad vinculada al dolor crónico orofacial.

2. La presencia de estas comorbilidades asociadas a una DTM contribuye a describir una peor situación clínica y funcional en estos pacientes, especialmente en cuanto desarrollo y percepción del dolor crónico.

3. Creemos que el papel de las comorbilidades asociadas a la DTM debe ser tenido en cuenta, ya que nos puede permitir realizar un nuevo enfoque integrado y amplio en un equipo multidisciplinar para una gestión terapéutica de la patología temporomandibular más adecuada.

\section{CONFLICTOS DE INTERESES}

Los autores declaran no tener ningún conflicto de intereses en la elaboración de este estudio.

\section{B I B L I O G R A F Í A}

1. Sato FRL, Lima CAA, Tralli G, da Silva RA. Is there a correlation between arthroscopic findings and the clinical signs and symptoms of patients with internal derangement of the temporomandibular joint? A prospective study. Int J Oral Maxillofac Surg. 2019;48(2):233-8. DOI: 10.1016/j.ijom.2018.07.004.

2. Muñoz-Guerra MF, Rodríguez-Campo FJ, Zylberberg I, DíazReverand S, Brabyn PJ, Fernández-Domínguez M, et al. Influ- 
encia de la edad en los hallazgos y el resultado del tratamiento artroscópico de la disfunción temporomandibular. Un estudio basado en 162 casos. Rev Esp Cir Oral Maxilofac. 2018;40(4):153-62. DOI: 10.1016/j.maxilo.2017.11.005.

3. Moreno-Fernández AM, Jiménez-Castellanos E, Iglesias-Linares A, Bueso-Madrid D, Fernández-Rodríguez A, de Miguel M. Fibromyalgia syndrome and temporomandibular disorders with muscular pain. A review. Mod Rheumatol. 2017;27(2):2106. DOI: 10.1080/14397595.2016.1221788.

4. Cho-Lee GY, Cho-Jung H, Castrejón-Castrejón S, Muñoz-Guerra MF, Rodríguez-Campo FJ, Díaz-González FJ, et al. Eficacia de la acupuntura en el tratamiento del síndrome de dolor miofascial de la musculatura masticatoria. Rev Esp Cir Oral Maxilofac. 2019;41(1):8-16. DOI: 10.20986/recom.2019.1027/2019.

5. Wilkes $\mathrm{CH}$. Internal derangements of the temporomandibular joint. Pathological variations. Arch Otolaryngol Head Neck Surg. 1989;115(4):469-77. DOI: 10.1001/archotol.1989.01860280067019.

6. Emshoff R, Brandlmaier I, Bertram S, Rudisch A. Relative odds of temporomandibular joint pain as a function of magnetic resonance imaging findings of internal derangement, osteoarthrosis, effusion, and bone marrow edema. Oral Surg Oral Med Oral Pathol Oral Radiol Endod. 2003;95(4):437-45. DOI: 10.1067/moe.2003.95.

7. Fernández-Ferro M, Fernández-Sanromán J, Blanco-Carrión A, Costas-López A, López-Betancourt A, Arenaz-Bua J, et al. Comparison of intra-articular injection of plasma rich in growth factors versus hyaluronic acid following arthroscopy in the treatment of temporomandibular dysfunction: A randomised prospective study. J Craniomaxillofac Surg. 2017;45(4):449-54. DOI: 10.1016/j.jcms.2017.01.010.

8. Ananthan S, Benoliel R. Chronic orofacial pain. J Neural Transm (Vienna). 2020;127(4):575-88. DOI: 10.1007/s00702-020-02157-3.

9. Fernández-de-las-Peñas C, Svensson P. Myofascial Temporomandibular Disorder. Curr Rheumatol Rev. 2016;12(1):40-54. DOI: 10.2174/1573397112666151231110947.

10. Costa YM, Conti PC, de Faria FA, Bonjardim LR. Temporomandibular disorders and painful comorbidities: clinical association and underlying mechanisms. Oral Surg Oral Med Oral Pathol Oral Radiol. 2017;123(3):288-97. DOI: 10.1016/j. oooo.2016.12.005.

11. Garrigós-Pedrón M, La Touche R, Navarro-Desentre P, GraciaNaya M, Segura-Ortí E. Effects of a Physical Therapy Protocol in Patients with Chronic Migraine and Temporomandibular Disorders: A Randomized, Single-Blinded, Clinical Trial. J Oral Facial Pain Headache. 2018;32(2):137-50. DOI: 10.11607/ ofph.1912.

12. Reiter S, Emodi-Perlman A, Goldsmith C, Friedman-Rubin P, Winocur E. Comorbidity between depression and anxiety in patients with temporomandibular disorders according to the research diagnostic criteria for temporomandibular disorders. J Oral Facial Pain Headache. 2015;29(2):135-43. DOI: 10.11607/ ofph.1297.

13. Dahan H, Shir Y, Velly A, Allison P. Specific and number of comorbidities are associated with increased levels of temporomandibular pain intensity and duration. J Headache Pain. 2015;16:528. DOI: 10.1186/s10194-015-0528-2.
14. Yunus MB. Central sensitivity syndromes: a new paradigm and group nosology for fibromyalgia and overlapping conditions, and the related issue of disease versus illness. Semin Arthritis Rheum. 2008;37(6):339-52. DOI: 10.1016/j.semarthrit.2007.09.003.

15. Schiffman E, Ohrbach R, Truelove E, Look J, Anderson G, Goulet JP, et al. International RDC/TMD Consortium Network, International association for Dental Research; Orofacial Pain Special Interest Group, International Association for the Study of Pain. Diagnostic Criteria for Temporomandibular Disorders (DC/TMD) for Clinical and Research Applications: recommendations of the International RDC/TMD Consortium Network* and Orofacial Pain Special Interest Group†. J Oral Facial Pain Headache. 2014;28(1):6-27. DOI: 10.11607/jop.1151.

16. Ayouni I, Chebbi R, Hela Z, Dhidah M. Comorbidity between fibromyalgia and temporomandibular disorders: a systematic review. Oral Surg Oral Med Oral Pathol Oral Radiol. 2019;128(1):33-42. DOI: 10.1016/j.00oo.2019.02.023.

17. Leblebici B, Pekta囚 ZO, Ortancil O, Hürcan EC, Bagis S, Akman MN. Coexistence of fibromyalgia, temporomandibular disorder, and masticatory myofascial pain syndromes. Rheumatol Int. 2007;27(6):541-4. DOI: 10.1007/s00296-006-0251-z.

18. Fraga BP, Santos EB, Farias Neto JP, Macieira JC, Quintans LJ Jr, et al. Signs and symptoms of temporomandibular dysfunction in fibromyalgic patients. J Craniofac Surg. 2012;23(2):615-8. DOI: 10.1097/SCS.0b013e31824cd81a.

19. Fenton BT, Goulet JL, Bair MJ, Cowley T, Kerns RD. Relationships Between Temporomandibular Disorders, MSD Conditions, and Mental Health Comorbidities: Findings from the Veterans Musculoskeletal Disorders Cohort. Pain Med. 2018;1;19(suppl_1):S61S68. DOI: 10.1093/pm/pny145.

20. González-Ballester D, González-García R, García-Nogales A, Moreno-García C, Monje-Gil F. ¿Es el desplazamiento discal sinónimo de patología temporomandibular? Correlación clínico-radiológica y prevalencia de trastornos internos en sujetos sanos asintomáticos. Rev Esp Cir Oral Maxilofac. 2020;42(3):97106. DOI:10.20986/recom.2020.1133/2020.

21. Maísa Soares G, Rizzatti-Barbosa CM. Chronicity factors of temporomandibular disorders: a critical review of the literature. Braz Oral Res. 2015;29:S1806-83242015000100300. DOI: 10.1590/1807-3107BOR-2015.vol29.0018.

22. Bertoli E, de Leeuw R. Prevalence of Suicidal Ideation, Depression, and Anxiety in Chronic Temporomandibular Disorder Patients. J Oral Facial Pain Headache. 2016;30(4):296-301. DOI: 10.11607/ofph.1675.

23. Arango-Dávila CA, Rincón-Hoyos HG. Depressive Disorder, Anxiety Disorder and Chronic Pain: Multiple Manifestations of a Common Clinical and Pathophysiological Core. Rev Colomb Psiquiatr.2018;47(1):46-55. DOI: 10.1016/j.rcp.2016.10.007.

24. Slade GD, Fillingim RB, Sanders AE, Bair E, Greenspan JD, Ohrbach R, Dubner R, Diatchenko L, Smith SB, Knott C, Maixner W. Summary of findings from the OPPERA prospective cohort study of incidence of first-onset temporomandibular disorder: implications and future directions. J Pain. 2013;14(12 Suppl):T116-24. DOI: 10.1016/j.jpain.2013.09.010.

25. Craske MG, Stein MB. Anxiety. Lancet. 2016;388(10063):3048-59. DOI: 10.1016/S0140-6736(16)30381-6. 